\title{
MODELS OF THE VENUS IONOSPHERE
}

\author{
J. R. HERMAN, R. E. HARTLE, and S. J. BAUER \\ Laboratory for Planetary Atmospheres, Goddard Space Flight Center, NASA, \\ Greenbelt, Md., U.S.A.
}

Theoretical modeling of the daytime Venus ionosphere can be used to augment the measurements of Mariner V made during the 1967 fly-by mission of Venus. The models discussed here are obtained by solving the equations of heat conduction for the electron, ion, and neutral gases along with the momentum and chemical equations for the charged particle densities [1, 2, 3]. When the model is brought into conformity with as much of the data as is possible, constraints can be placed on some of the unknown parameters such as the electron and ion temperatures, and the strength of the magnetic field in the topside Venus ionosphere.

Most of the boundary conditions needed to construct the ionosphere models can be obtained directly from the Mariner V measurements [4]. The upper boundary is selected to be at the altitude of the observed abrupt termination of the electron density profile near $500 \mathrm{~km}$. Since the peak electron density of $5.2 \times 10^{5} \mathrm{~cm}^{-3}$ occurs at an altitude of 135 to $140 \mathrm{~km}$, the lower boundary, or reference altitude, is placed at $100 \mathrm{~km}$. It has been suggested by several authors that the abrupt termination of the electron density profile in the topside ionosphere, the ionopause, arises from the fact that the magnetic fields carried along by the solar wind are forced to pile up on top of the highly conducting ionosphere. This magnetic obstacle then forms a natural upper boundary for the ionosphere and interacts with the super-alfvenic solar wind to form a bow shock that has been observed at about $50000 \mathrm{~km}$ from Venus.

If the momentum and energy equations are applied across the bow shock, the resulting density just within the bow shock is about $12 \mathrm{~cm}^{-3}$ with a corresponding proton temperature of approximately $4 \times 10^{6} \mathrm{~K}$. Near the ionopause these values can be used to obtain a pressure balance that requires a magnetic field build up to about $50 \gamma$. Since the ionopause is interpreted as the interface between the solar wind and the Venus ionosphere, a balance must be made between the total solar wind pressure $P_{w}$ and the total charged particle pressure $P_{c}$ immediately below the ionopause. This pressure balance, $P_{c}=P_{w} \cong K N M V^{2} \cos ^{2} \psi$, calculated at $\psi=45^{\circ}$ from the subsolar wind point, forms one of the boundary conditions for our model. The precise value of the pressure depends upon the value of the accomodation coefficient $K$ [5]. Since the most likely value lies near one, we have adopted $K=1$ to obtain $P_{c}=8.78 \times 10^{-9}$ dyne-cm ${ }^{-2}$ for a solar wind velocity $V=590 \mathrm{~km} / \mathrm{sec}$ and a density of $N=3 \mathrm{~cm}^{-3}$. Figure 1 illustrates this schematically.

The presence or absence of a magnetic field has important consequences for the thermal structure of the charged particles. There are two extremes: (1) where the presence of an essentially horizontal magnetic field inhibits thermal conduction across field lines and (2) where due to the complete absence of a magnetic field or to the presence of a tilted magnetic field and possible turbulence, the thermal structure is

Sagan et al. (eds.), Planetary Atmosphere's, 23-27.

All Rights Reserted. Copyright o 1971 by the I.A.l 


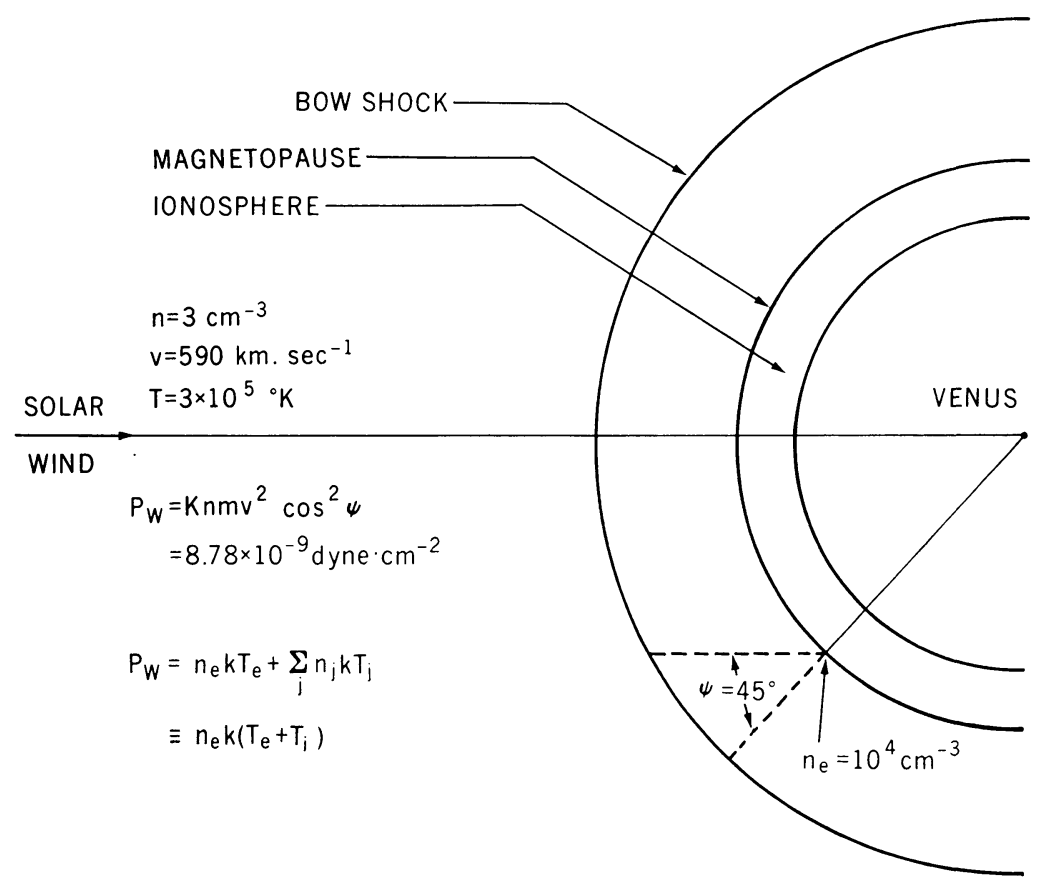

Fig. 1. Schematic representation of the solar wind interaction with the Venus ionosphere.

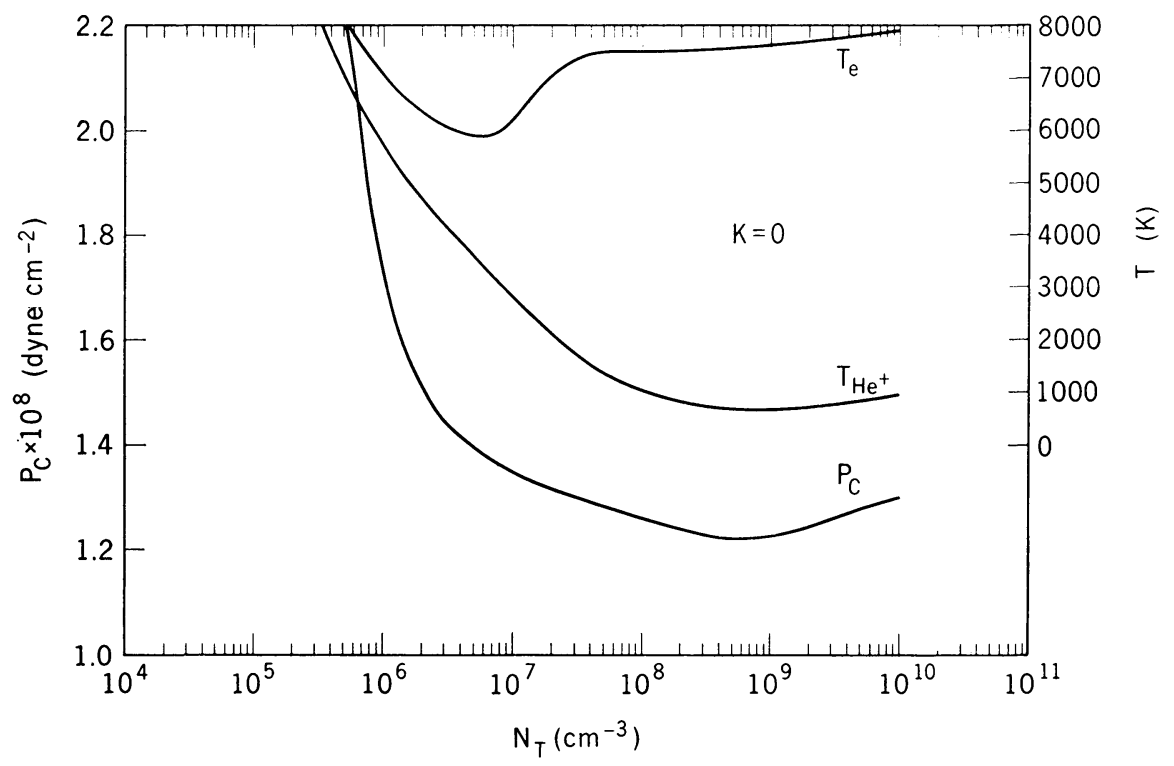

Fig. 2. The charged particle pressure $P_{c}$, electron temperature $T_{e}$, and major ion temperature $T_{\mathrm{Hc}^{+}}$just below the ionopause as a function of the neutral helium concentration $N_{T}$ at the reference altitude $100 \mathrm{~km}$. The thermal conductivity $K=0$. 


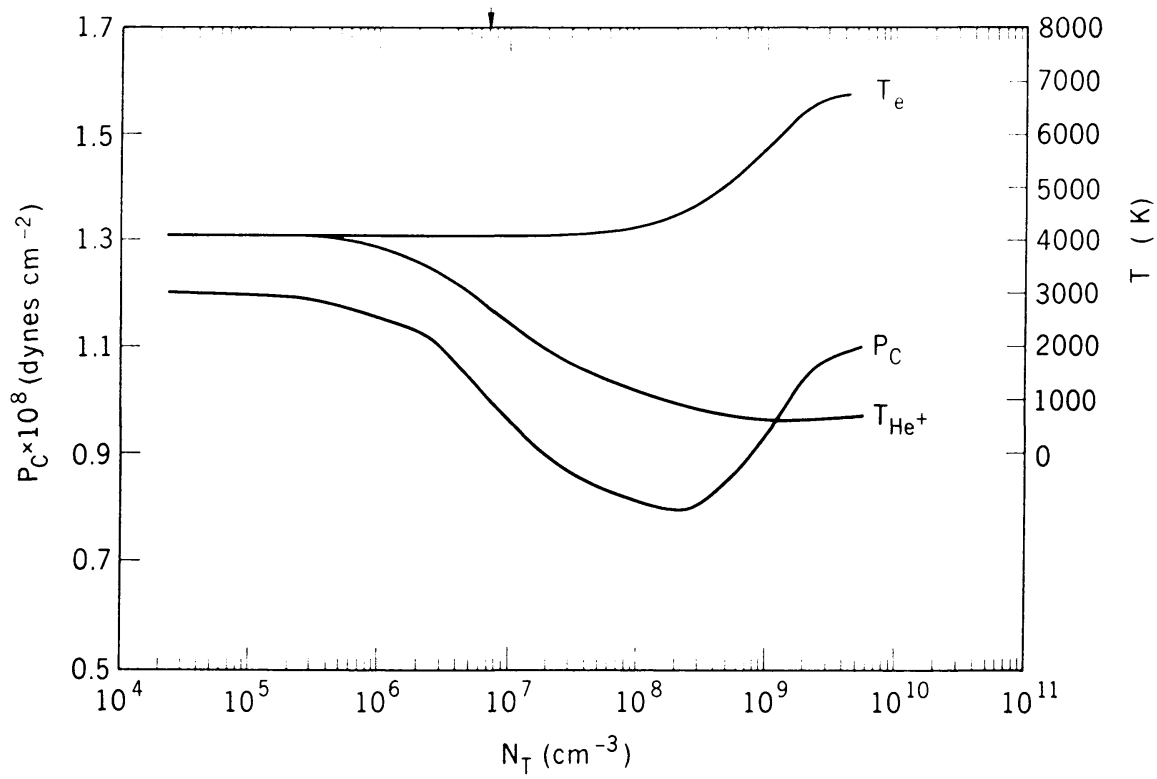

Fig. 3. The same conditions as in Figure 2, except $K=0.006 K_{\|}$.

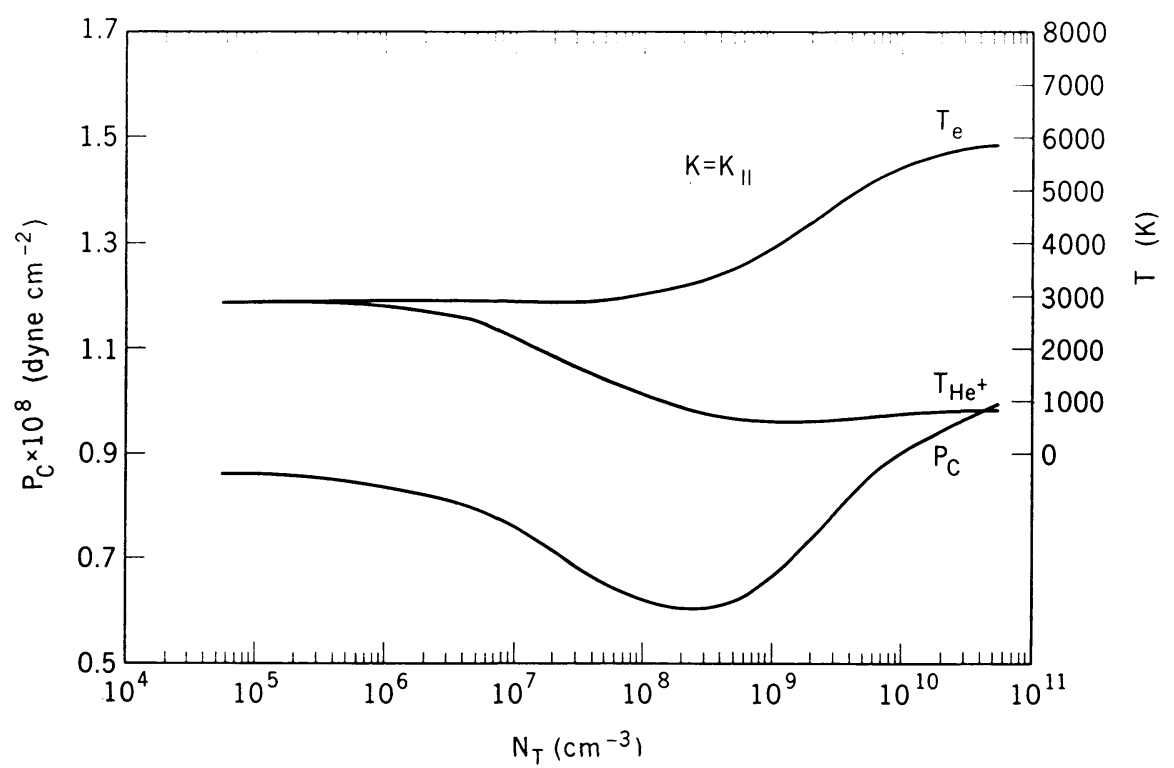

Fig. 4. The same conditions as in Figure 2, except $K=K_{\text {ii }}$. 


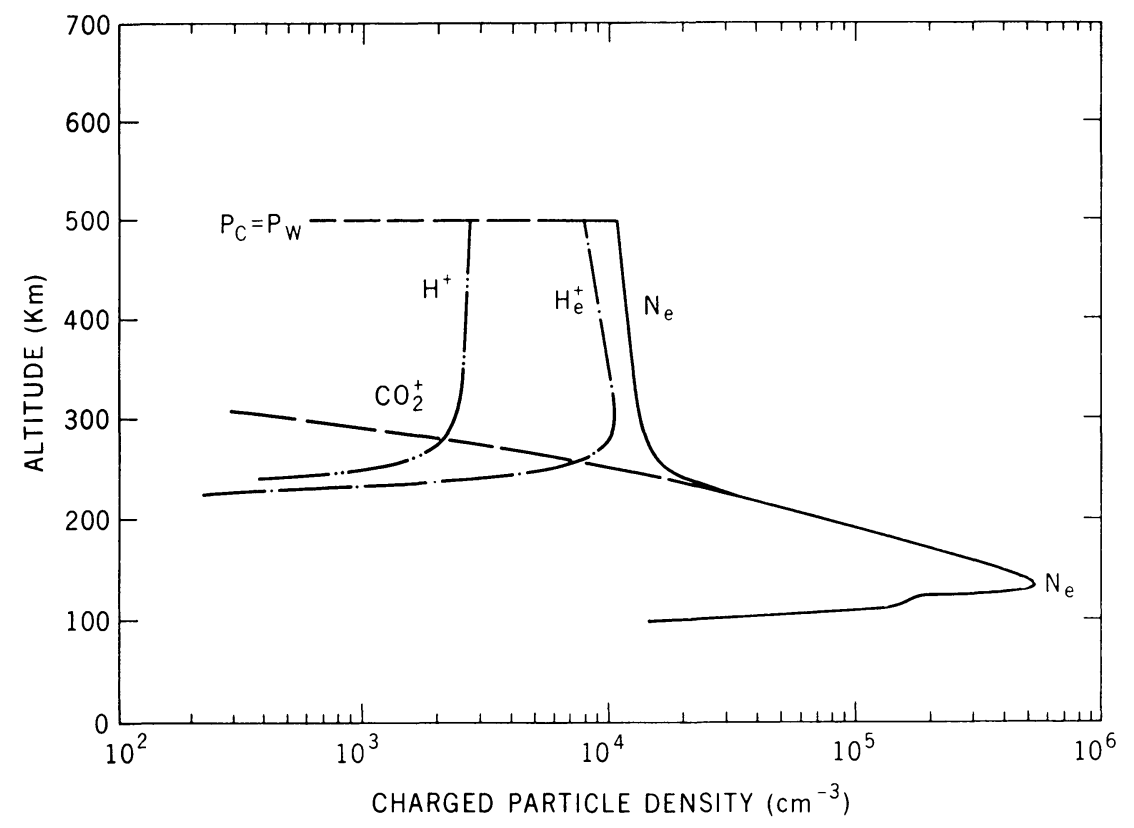

(a)

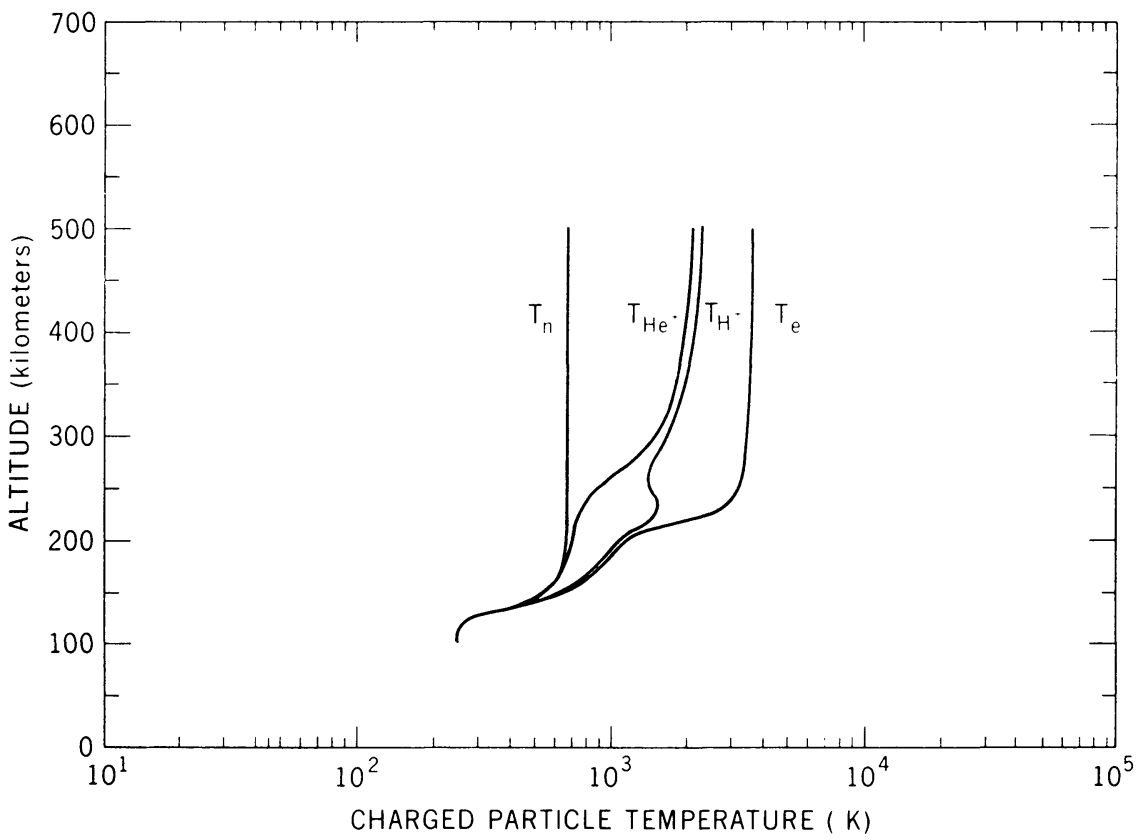

(b)

Fig. 5a, b. The electron, ion, and neutral gas temperatures, and the ion composition for boundary conditions compatible with the required pressure balance, $P_{c}=P_{w} \cong K N M V^{2} \cos ^{2} \psi$, and when $K=0.006 K_{!}$. At $100 \mathrm{~km}\left(\mathrm{~N}_{2}\right)=5 \times 10^{8},\left(\mathrm{CO}_{2}\right)=2 \times 10^{13},\left(\mathrm{H}_{2}\right)=4 \times 10^{6},(\mathrm{He})=5 \times 10^{7},(\mathrm{H})=2 \times 10^{4}$, (D) $=1 \times 10^{6}$, with a hydrostatic distribution above $100 \mathrm{~km}$. 
strongly controlled by thermal conduction. If a series of solutions are constructed as shown in Figures 2, 3, and 4, the density of neutral helium $N_{T}$ at $100 \mathrm{~km}$, the strength of the magnetic field within the ionosphere, and the effective tilt* (in terms of magnetic dip angle) can be estimated. Since a magnetic field within the ionosphere exerts a pressure $B^{2} / 8 \pi$, the charged particle pressure need only make up the difference to balance the solar wind pressure. For example, for fields of 10, 20, and $30 \gamma, P_{c}$ (500 $\mathrm{km})$ must be $8.4 \times 10^{-9}, 7.2 \times 10^{-9}$, and $5.2 \times 10^{-9}$ dyne-cm ${ }^{-2}$ respectively. As can be seen from the figures, no solutions exist for dip angles less than about $3.5^{\circ}$, which is equivalent to the thermal conductivity $K$ less than $0.0037 K_{\|}$. Because of the essentially horizontal nature of the induced magnetic field and the small upper limit placed on a possible intrinsic planetary magnetic moment [6], large effective dip angles are not possible. Thus, the magnetic field within the ionosphere has a strength between 10 and $20 \gamma$ with an effective magnetic dip angle near $4.5^{\circ}\left(K=0.006 K_{\|}\right)$. For these values, the neutral helium density at $100 \mathrm{~km}$ must lie in the range $3 \times 10^{7}$ to $6 \times 10^{8} \mathrm{~cm}^{-3}$ as can be seen from Figure 3.

Selecting boundary values corresponding to the acceptable range of solutions in Figure 3, the thermal structure and ion composition of the daytime Venus ionosphere can be obtained. The solutions shown in Figure 5 predict that the electron temperature $T_{e}$ and the major ion temperature $T_{\mathrm{He}^{+}}$are not in thermal equilibrium with the neutral gas temperature $T_{n}$ except at altitudes near $100 \mathrm{~km}$. In the region near $500 \mathrm{~km}$, $T_{e}=3700 \mathrm{~K}, T_{\mathrm{He}^{+}}=2100 \mathrm{~K}$, and $T_{n}=660 \mathrm{~K}$.

\section{References}

[1] Herman, J. R. and Chandra, S.: 1969, Planetary Space Sci. 17, 815.

[2] Bauer, S. J., Hartle, R. E., and Herman, J. R.: 1970, Nature 225, 533.

[3] McElroy, M. B.: 1969, J. Geophys. Res. 74, 29.

[4] Kliore, A., Levy, G., Cain, D., Fjeldbo, G., and Rasool, S.: 1967, Science 158, 1683.

[5] Schield, M. A.: 1969, J. Geophys. Res. 74, 1275.

[6] Dolginov, S. S., Yeroshenko, E. G., and Zhuzgov, L. N.: 1968, Kosm. Issled. Moscow. NASA Translation ST-LPS-PMF-10730.

* The effective magnetic dip angle may be composed in part of: (1) an actual geometrical tilt derived from the sum of the intrinsic and induced fields; (2) bulk motion of the high $\beta$ Venus ionosphere that produces significant distortion in the magnetic field; (3) the possible presence of turbulence that can change the effective thermal conductivity $K=\Omega K_{\|}$. 